\title{
Therapeutic drug monitoring to prevent acquired drug resistance of fluoroquinolones in the treatment of tuberculosis
}

To the Editor:

The new short-course World Health Organization (WHO)-endorsed regimen for multidrug-resistant (MDR) tuberculosis (TB) resistant to rifampicin and isoniazid gives hope for patients and care-givers. However, there is still an unacceptable proportion of treatment failure and evidence of man-made acquired drug resistance of fluoroquinolones during programmatic treatment of MDR-TB [1]. The appropriate use of fluoroquinolones is crucial in MDR-TB treatment, perhaps even more so in the new WHO-endorsed short-course MDR-TB regimen. Current shortcomings with inappropriate use of fluoroquinolones, such as underdosing, need to be avoided in order to prevent failure of the new short-course regimen.

The high failure rates of MDR-TB patients may partly be due to a mismatch in drug exposure and bacterial susceptibility [2]. In many settings, this mismatch is neglected, due to lack of awareness, laboratory capacity or resources.

FALzon et al. [3] recently described the current problems with the fluoroquinolone use for MDR-TB and the search for implementable measures. In addition, we would like to introduce therapeutic drug monitoring (TDM) of fluoroquinolones as a potential strategy for optimising drug exposure of fluoroquinolones in daily clinical practice.

Low drug exposure is commonly seen and may induce drug resistance and treatment failure. In addition to adherence, pharmacokinetic between-patient variability is an important factor that influences drug exposure $[4,5]$. In a hollow-fibre model mimicking human drug concentrations, resistance to fluoroquinolones occurred in $40 \%$ of cases when $400 \mathrm{mg}$ moxifloxacin was used with Mycobacterium tuberculosis isolates with minimum inhibitory concentrations (MICs) of $0.25 \mathrm{mg} \cdot \mathrm{L}^{-1}$ [2]. This phenomenon has also been illustrated in a prospective cohort of 832 patients, in which $12.1 \%$ of patients acquired fluoroquinolone resistance [1].

It is obvious that adequate drug concentrations in $\mathrm{TB}$ patients are needed to kill bacteria. For moxifloxacin, a nine-fold variability in drug exposure was observed between patients receiving the same dose [5], mainly due to between-patient variability in pharmacokinetic parameters. Measurement of actual drug exposure is especially important in patients with comorbidities like HIV, type 2 diabetes or conditions related to malabsorption [6]. Adequate drug concentrations can be ensured by TDM, enabling dose adjustments guided by drug concentrations and MICs [7].

Drug resistance cannot simply be proven or rejected by testing one drug concentration; rather does the level of drug resistance matter. Resistance in M. tuberculosis occurs through spontaneous mutations in the genome, but different mutations give rise to different levels of resistance.

Detection of resistance has been simplified by rapid molecular testing and the use of customised susceptibility plates to determine MICs. The most common mutations related to fluoroquinolone resistance can rapidly be detected by line probe assay (LPA). Unlike phenotypic drug susceptibility testing, the MIC will provide a level of resistance, rather than just identifying resistance. Simple methods such as MYCOTB ${ }^{\mathrm{TM}}$ and Sensititre ${ }^{\mathrm{TM}}$ TREK enable simultaneous MIC testing of multiple antibiotics. The MYCOTB method was particularly sensitive in detecting fluoroquinolone resistance (91-98\%) in a recent study [8].

@ERSpublications

Therapeutic drug monitoring of fluoroquinolones might prevent acquired XDR-TB http://ow.ly/jj6b309QXyZ

Cite this article as: Davies Forsman L, Bruchfeld J, Alffenaar J-WC. Therapeutic drug monitoring to prevent acquired drug resistance of fluoroquinolones in the treatment of tuberculosis. Eur Respir J 2017; 49: 1700173 [https://doi.org/10.1183/13993003.00173-2017]. 




FIGURE 1 The implementation of therapeutic drug monitoring for fluoroquinolones. When multidrug-resistant tuberculosis (MDR-TB) is diagnosed, further resistance, such as extensively drug-resistant tuberculosis (XDR-TB), needs to be excluded. Line probe assays will provide fast results, but should be confirmed by phenotypic drug susceptibility testing (DST). When XDR-TB excluded, a fluoroquinolone-containing treatment regimen can be initiated. In order to avoid low drug concentrations, a minimum inhibitory concentration $(\mathrm{MIC})$ might be determined, simplified by MycoTB $®$ or Sensititre TREK®. After two weeks of treatment, multiple drug concentrations of fluoroquinolones can be measured using 2-3 blood samples and drug exposure estimated by the area under the curve (AUC) versus time can be determined. The drug exposure should then be compared with the MIC of the bacteria and dose adjusted if needed, with an optimal ratio of AUC/MIC $>100$. PK: pharmacokinetics; PD: pharmacodynamics.

Drug exposure should therefore be viewed in relation to the level of resistance of the bacteria, to ensure efficacy. The area under the concentration time curve (AUC)/MIC for fluoroquinolones is a predictor of clinical outcome, with suggested target levels of AUC/MIC of $>100[2,9]$. However, at MICs near the critical breakpoint, the target AUC/MIC ratios are often not reached. Therefore, experts recommend TDM for MICs $\geqslant 1 \mathrm{mg} \cdot \mathrm{L}^{-1}$ of levofloxacin and $\geqslant 0.25 \mathrm{mg} \cdot \mathrm{L}^{-1}$ of moxifloxacin $[5,10]$.

If the AUC/MIC ratio justifies a dose increase, patient safety is important. Careful monitoring of ECGs should be performed in patients receiving moxifloxacin with other risk factors of QT-prolongation, such as hypokalaemia. Nevertheless, doses up to $800 \mathrm{mg}$ moxifloxacin have been found safe in clinical trials. The STREAM study will provide additional information about the safety of high dose fluoroquinolones.

Nowadays, TDM can be implemented, even in resource-limited settings. To minimise the number of blood samples needed to evaluate drug exposure, limited sampling strategies have been developed. Collection of a drop of blood on dried blood spot cards that are easily stored and transported enables TDM even in rural settings [7]. If MICs are high, drug concentrations should be determined and vice versa, if drug concentrations are low, an MIC will reveal if a sufficient AUC/MIC ratio is reached. Figure 1 provides a suggested flow chart for clinical practice.

In conclusion, fluoroquinolones are at risk of acquired drug resistance despite multidrug regimens and good adherence. An intervention is urgently needed, especially for the WHO recommended short course regimen. TDM can be performed under programmatic conditions in various centres in the world and is no longer limited to resource-rich settings [11]. Thus, TDM for fluoroquinolones in MDR-TB treatment could ensure sufficient drug exposure, minimise further drug resistance development and improve treatment outcome.

Lina Davies Forsman ${ }^{1,2}$, Judith Bruchfeld ${ }^{1,2}$ and Jan-Willem C. Alffenaar ${ }^{3}$

${ }^{1}$ Karolinska Institutet, Dept of Medicine, Unit of Infectious Disease, Stockholm, Sweden. ${ }^{2}$ Karolinska University Hospital, Dept of Infectious Disease, Stockholm, Sweden. ${ }^{3}$ University of Groningen, University Medical Center Groningen, Dept of Clinical Pharmacy and Pharmacology, Groningen, The Netherlands. 
Correspondence: J.W.C. Alffenaar, University Medical Center Groningen, Dept of Clinical Pharmacy and Pharmacology, PO box 30.001, 9700 RB Groningen, The Netherlands. E-mail: j.w.c.alffenaar@umcg.nl

Received: Jan 242017 | Accepted after revision: Feb 152017

Conflict of interest: None declared.

\section{References}

1 Cegielski JP, Dalton T, Yagui M, et al. Extensive drug resistance acquired during treatment of multidrug-resistant tuberculosis. Clin Infect Dis 2014; 59: 1049-1063.

2 Gumbo T, Louie A, Deziel MR, et al. Selection of a moxifloxacin dose that suppresses drug resistance in Mycobacterium tuberculosis, by use of an in vitro pharmacodynamic infection model and mathematical modeling. J Infect Dis 2004; 190: 1642-1651.

3 Falzon D, Schünemann HJ, Harausz E, et al. World Health Organization treatment guidelines for drug-resistant tuberculosis, 2016 update. Eur Respir J 2017; 49: 1602308.

4 Srivastava S, Pasipanodya JG, Meek C, et al. Multidrug-resistant tuberculosis not due to noncompliance but to between-patient pharmacokinetic variability. J Infect Dis 2011; 204: 1951-1959.

5 Pranger $\mathrm{AD}$, van Altena R, Aarnoutse RE, et al. Evaluation of moxifloxacin for the treatment of tuberculosis: 3 years of experience. Eur Respir J 2011; 38: 888-894.

6 Nahid P, Dorman SE, Alipanah N, et al. Official American Thoracic Society/Centers for Disease Control and Prevention/Infectious Diseases Society of America Clinical Practice Guidelines: treatment of drug-susceptible tuberculosis. Clin Infect Dis 2016; 63: e147-e195.

7 Ghimire S, Bolhuis MS, Sturkenboom MG, et al. Incorporating therapeutic drug monitoring into the World Health Organization hierarchy of tuberculosis diagnostics. Eur Respir J 2016; 47: 1867-1869.

8 Foongladda S, Banu S, Pholwat S, et al. Comparison of TaqMan ${ }^{\star}$ Array Card and MYCOTB ${ }^{\mathrm{TM}}$ with conventional phenotypic susceptibility testing in MDR-TB. Int J Tuberc Lung Dis 2016; 20: 1105-1112.

9 Mpagama SG, Ndusilo N, Stroup S, et al. Plasma drug activity in patients on treatment for multidrug-resistant tuberculosis. Antimicrob Agents Chemother 2014; 58: 782-788.

10 Ghimire S, Van't Boveneind-Vrubleuskaya N, Akkerman OW, et al. Pharmacokinetic/pharmacodynamic-based optimization of levofloxacin administration in the treatment of MDR-TB. J Antimicrob Chemother 2016; 71: 2691-2703.

11 Alffenaar JC, Tiberi S, Verbeeck RK, et al. Therapeutic drug monitoring in tuberculosis: practical application for physicians. Clin Infect Dis 2017; 64: 104-105.

Copyright @ERS 2017

From the authors:

Later-generation fluoroquinolones (moxifloxacin, gatifloxacin) are crucial to all treatment regimens in current use for multidrug-resistant (MDR) tuberculosis (TB) (i.e. joint resistance to at least rifampicin and isoniazid) [1]. Resistance to them is known to occur in different geographical settings [2]. L. Davies Forsman and co-workers advocate for therapeutic drug monitoring (TDM) as a means to help keep blood levels of fluoroquinolones within the ranges considered effective during MDR-TB treatment. They refer to reports of substantial variability in fluoroquinolone pharmacokinetics between patients, which makes it difficult to predict when sub-therapeutic levels occur, and could thus predispose to treatment failure and to the emergence of resistant strains. The authors maintain that TDM employing a dried blood spot technique and newer microbroth dilution plate-based methods to test for minimum inhibitory concentration (MICs) now make it possible for clinicians in low-resource settings to ensure that therapeutic levels of fluoroquinolones can be achieved during treatment.

While this reasoning is mainstream and logical and has already been expressed in other articles featured in this journal [3], a number of uncertainties stand in the way of wide recommendations for their use. The correlation between tissue concentration of fluoroquinolones and clinical outcomes is still based on small numbers of observations of patients on treatment. The rapid tests for MIC to multiple antimicrobials cited by the authors still require validation. The feasibility of implementing these tests and TDM under routine programmatic practice still needs to be shown, especially in high TB burden and resource-constrained contexts.

@ERSpublications

Could therapeutic drug monitoring for fluoroquinolones have a role in the programmatic management of MDR-TB? http://ow.ly/wGdP309R7Z4

Cite this article as: Falzon D, Jaramillo E, Gilpin C, et al. Therapeutic drug monitoring to prevent acquired drug resistance of fluoroquinolones in the treatment of tuberculosis. Eur Respir J 2017; 49: 1700317 [https:// doi.org/10.1183/13993003.00317-2017]. 
Regardless of this, no effort should be spared to forestall the development of resistance to fluoroquinolones during treatment, a known correlate with poorer patient outcomes [4]. Only about one half of MDR-TB patients worldwide are known to complete their treatment successfully [5]. While about 580000 new patients requiring MDR-TB treatment are estimated to emerge each year, only about 125000 patients were reported to have been started on second-line TB treatment in 2015. Since 2016, the World Health Organization (WHO) recommends a shorter MDR-TB regimen with a composition and dosing schedule identical to that used in a randomised controlled trial, including high-dose moxifloxacin [1,6-8]. This shorter regimen is expected to facilitate the expansion of treatment to more patients and to improve outcomes in those who are eligible. Prior exposure or confirmed resistance to fluoroquinolones, which could favour the acquisition of additional drug-resistance, is among the contraindications to this regimen. Clinical monitoring for response to treatment and for potential harms related to medication (e.g. electrocardiography, serum potassium) is recommended in patients on any novel regimen [9]. Well conducted operational research using observational cohorts could be valuable if blinded randomised controlled trials do not materialise in future. Considerations on whether or when to add TDM to tests generally advised in MDR-TB patients will be discussed at a technical consultation being convened by WHO in April 2017, which will look at critical concentrations and pharmacokinetics/pharmacodynamics of TB medicines.

Dennis Falzon, Ernesto Jaramillo, Christopher Gilpin and Karin Weyer

Global TB Programme, World Health Organization (WHO), Geneva, Switzerland.

Correspondence: Dennis Falzon, Global TB Programme, World Health Organization (WHO), 20, Avenue Appia, CH-1211 Geneva, Switzerland. E-mail: falzond@who.int

Received: Feb 142017 | Accepted after revision: March 082017

Conflict of interest: None declared.

Acknowledgements: Authors are staff members of the World Health Organization (WHO). They alone are responsible for the views expressed in this publication and they do not necessarily represent the decisions or policies of WHO. The designations used and the presentation of the material in this publication do not imply the expression of any opinion whatsoever on the part of WHO concerning the legal status of any country, territory, city or area, or of its authorities, nor concerning the delimitation of its frontiers or boundaries.

\section{References}

1 World Health Organization. WHO treatment guidelines for drug-resistant tuberculosis, 2016 update (WHO/HTM/ TB/2016.04). Geneva, World Health Organization, 2016. Available from: www.who.int/tb/areas-of-work/ drug-resistant-tb/treatment/resources/en/

2 Zignol M, Dean AS, Alikhanova N, et al. Population-based resistance of Mycobacterium tuberculosis isolates to pyrazinamide and fluoroquinolones: results from a multicountry surveillance project. Lancet Infect Dis 2016; 16: 1185-1192.

3 Srivastava S, Peloquin CA, Sotgiu G, et al. Therapeutic drug management: is it the future of multidrug-resistant tuberculosis treatment? Eur Respir J 2013; 42: 1449-1453.

4 Falzon D, Gandhi N, Migliori GB, et al. Resistance to fluoroquinolones and second-line injectable drugs: impact on MDR-TB outcomes. Eur Respir J 2013; 42: 156-168.

5 World Health Organization. Global tuberculosis report 2016 (WHO/HTM/TB/2016.13). Geneva, World Health Organization, 2016. Available from: www.who.int/tb/publications/global_report/en/

6 World Health Organization. Frequently asked questions about the shorter MDR-TB regimen. www.who.int/entity/ tb/areas-of-work/drug-resistant-tb/treatment/FAQshorter_MDR_regimen.pdf Date last accessed: March 8, 2017.

7 Nunn AJ, Rusen ID, Van Deun A, et al. Evaluation of a standardized treatment regimen of anti-tuberculosis drugs for patients with multi-drug-resistant tuberculosis (STREAM): study protocol for a randomized controlled trial. Trials 2014; 15: 353.

8 Sotgiu G, Tiberi S, D’Ambrosio L, et al. Faster for less: the new 'shorter' regimen for multidrug-resistant tuberculosis. Eur Respir J 2016; 48: 1503-1507.

9 World Health Organization. Active tuberculosis drug-safety monitoring and management (aDSM). Framework for implementation. Geneva, World Health Organization, 2015. Available from: http://apps.who.int/iris/bitstream/ 10665/204465/1/WHO_HTM_TB_2015.28_eng.pdf 